\title{
Identification of Amino Acid Residues of the NR2A Subunit That Control Glutamate Potency in Recombinant NR1/NR2A NMDA Receptors
}

\author{
Lesley C. Anson, ${ }^{1,2}$ Philip E. Chen, ${ }^{1,2}$ David J. A. Wyllie, ${ }^{2}$ David Colquhoun,, ${ }^{1,2}$ and Ralf Schoepfer ${ }^{1,2}$ \\ University College London, 'Wellcome Laboratory for Molecular Pharmacology, and ${ }^{2}$ Department of Pharmacology, \\ London WC1E 6BT, United Kingdom
}

\begin{abstract}
The NMDA type of ligand-gated glutamate receptor requires the presence of both glutamate and glycine for gating. These receptors are hetero-oligomers of NR1 and NR2 subunits. Previously it was thought that the binding sites for glycine and glutamate were formed by residues on the NR1 subunit. Indeed, it has been shown that the effects of glycine are controlled by residues on the NR1 subunit, and a "Venus flytrap" model for the glycine binding site has been suggested by analogy with bacterial periplasmic amino acid binding proteins. By analysis of 10 mutant NMDA receptors, we now show that residues on the NR2A subunit control glutamate potency in recombinant NR1/NR2A receptors, without affecting glycine potency. Furthermore, we provide evidence that, at least for some mutated residues, the reduced potency of glutamate
\end{abstract}

cannot be explained by alteration of gating but has to be caused primarily by impairing the binding of the agonist to the resting state of the receptor. One NR2A mutant, NR2A(T671A), had an $E C_{50}$ for glutamate 1000 -fold greater than wild type and a 255-fold reduced affinity for APV, yet it had single-channel openings very similar to those of wild type. Therefore we propose that the glutamate binding site is located on NR2 subunits and (taking our data together with previous work) is not on the NR1 subunit. Our data further imply that each NMDA receptor subunit possesses a binding site for an agonist (glutamate or glycine).

Key words: N-methyl-D-aspartate (NMDA); glutamate; receptor; recombinant; subunit; ligand; binding; gating; single-channel; APV; Schild plot; affinity; oocyte; mutant; kinetic
The NMDA type of glutamate receptor forms a subfamily of ionotropic receptors with distinctive functional and biophysical properties. NMDA receptors play an important role in synaptic plasticity, memory and learning, brain development and excitotoxicity (for review, see Choi, 1988; McBain and Mayer, 1994; Collingridge and Bliss, 1995). Molecular cloning has identified a family of genes that code for subunits of ionotropic glutamate receptors (for review, see Nakanishi, 1992; Hollmann and Heinemann, 1994). One subfamily, GluR1-4, codes for the AMPA type of glutamate receptors; five other genes (NR1 and NR2A-D) code for NMDA receptor subunits.

NMDA receptors are endowed with a set of properties that distinguish them from other ionotropic glutamate receptors. Magnesium ions block the NMDA receptor channel at the resting potential, and current flows only when the synaptic release of agonist coincides with depolarization, so the receptor may act as a coincidence detector. NMDA receptor channels show a high permeability for calcium ions, the influx of which can trigger important cellular processes.

In the context of this paper two features are of special interest. (1) Both glutamate and glycine have to be present for efficient gating of the NMDA receptor channel. This requirement for a

Received Aug. 25, 1997; revised Oct. 23, 1997; accepted Oct. 24, 1997.

This work was funded by the Wellcome Trust. R.S. is a Senior Wellcome Trust Fellow. P.E.C. is supported by a Medical Research Council studentship. D.J.A.W. is a Royal Society University Research Fellow. We thank Peter Seeburg for NMDA receptor subunit cDNA and Philippe Béhé for unpublished data on wild-type receptor.

Correspondence should be addressed to Dr. Ralf Schoepfer, University College London, Department of Pharmacology, Wellcome Laboratory for Molecular Pharmacology, London WC1E 6BT, UK.

Copyright (c) 1998 Society for Neuroscience $\quad 0270-6474 / 98 / 180581-09 \$ 05.00 / 0$ coagonist is in contrast to all other known glutamate receptors. (2) NMDA receptors are hetero-oligomers that are composed of at least two types of subunit: the constant NR1 subunit, which comes in multiple splice variants, and one or more types of an NR2 subunit of which four (NR2A-D) variants are known. This is in contrast to the recombinant AMPA type of glutamate receptors, which can be either homo-oligomers or heterooligomers of GluR1-4 subunits.

After the initial cloning of the NR1 subunit (Moriyoshi et al., 1991), it was suggested that the binding sites for glutamate and glycine are located on the NR1 subunit. Indeed, site-directed mutagenesis of the NR1 subunit has identified amino acid residues that control glycine potency (Kuryatov et al., 1994; Wafford et al., 1995; Hirai et al., 1996; Williams et al., 1996). In these studies, single amino acid mutations reduced glycine potency by up to four orders of magnitude, with only small effects on glutamate potency. These studies suggest further that the glycine binding pocket has a bi-lobar structure, similar to that found by crystallography for a family of bacterial periplasmic amino acid binding proteins (Oh et al., 1993, 1994), in which the ligand is bound in a "Venus flytrap" mechanism.

Here we report the identification of amino acids, through site-directed mutagenesis, that control glutamate potency in recombinant NR1/NR2A receptors. These residues were found on the NR2A subunit, at positions homologous to those on the NR1 subunit that control glycine potency. Similar conclusions were published recently by Laube et al. (1997). Furthermore, we now provide evidence that at least some of these residues shape the glutamate binding site. Thus, the overall folding of the area of NR2 subunits that binds glutamate is likely to be structurally similar to the area of the NR1 subunit that binds glycine. 


\section{MATERIALS AND METHODS}

Expression plasmid constructs. The wild-type expression plasmids for the rat NR1 (1a splice form) and NR2A NMDA receptor subunits were as in Kuner and Schoepfer (1996). Mutations were introduced into the NR2A sequence by a PCR-based strategy. To facilitate cloning and identification of mutants, some NR2A mutants carried additional silent nucleotide substitutions. PCR-generated DNA segments and cloning sites were confirmed by DNA sequencing.

Heterologous expression of $N M D A$ receptors. cRNA was synthesized as runoff transcripts from linearized plasmid DNA using the Promega (Madison WI) RiboMax RNA synthesis kit. Reactions were supplemented with $0.75 \mathrm{~mm}$ capping nucleotide $\mathrm{m}^{7} \mathrm{G}\left(5^{\prime}\right) \mathrm{ppp}\left(5^{\prime}\right) \mathrm{G}$ (Pharmacia, Uppsala, Sweden) in the presence of $1.6 \mathrm{~mm}$ GTP. cRNA amounts and integrity were estimated by intensity of fluorescence in ethidium bromide-stained agarose gels, and concentration was determined also by $\mathrm{OD}_{260}$. NR1 and NR2A cRNAs were mixed at a nominal ratio of 1:1 and diluted with water to $\sim 5 \mathrm{ng} / \mu$ l of each, before injection. Xenopus laevis oocytes (stage V) were isolated manually after collagenase treatment (Sigma type V, $30 \mu \mathrm{g} / \mathrm{ml}$, at room temperature for $1 \mathrm{hr}$ ). Oocytes were then defolliculated and injected (Drummond Injector) with $23 \mathrm{nl}$ of cRNA mixture. For expression the oocytes were incubated at $19^{\circ} \mathrm{C}$ for 2-3 d in modified Barth's solution (in mM): $\mathrm{NaCl} 88, \mathrm{KCl} 1, \mathrm{NaHCO}_{3}$ $2.4, \mathrm{Ca}\left(\mathrm{NO}_{3}\right)_{2} 0.33, \mathrm{MgSO}_{4} 0.82, \mathrm{CaCl}_{2} 0.44$, Tris- $\mathrm{Cl} 15$, adjusted to $\mathrm{pH}$ 7.4 with $\mathrm{NaOH}$, supplemented with $50 \mathrm{IU} / \mathrm{ml}$ penicillin (BRL, Bethesda, $\mathrm{MD}$ ), $50 \mu \mathrm{g} / \mathrm{ml}$ streptomycin (BRL), and $30 \mu \mathrm{M} \mathrm{D}(-)-2$-amino-5phosphonopentanoic acid (APV) (Tocris), followed by storage at $4^{\circ} \mathrm{C}$ until they were used for electrophysiological measurements.

Whole-oocyte recording. Responses to glutamate were measured with a two-electrode voltage-clamp amplifier (TEC05, NPI electronics, Tamm, Germany) at $-60 \mathrm{mV}$ using $0.5-2.0 \mathrm{M} \Omega$ electrodes filled with $3 \mathrm{M} \mathrm{KCl}$. Oocytes were perfused with nominally $\mathrm{Ca}^{2+}$-free, low- $\mathrm{Ba}^{2+} \mathrm{NFR}$ solution (in $\mathrm{mm}$ ): $\mathrm{NaCl} 115, \mathrm{KCl} 2.5, \mathrm{HEPES} \mathrm{10}$, adjusted to $\mathrm{pH} 7.2$ with $\mathrm{HCl}$ and supplemented with $0.18 \mathrm{~mm} \mathrm{BaCl}_{2}$ unless noted otherwise.

Glutamate stock solutions $\left(1 \mathrm{M}\right.$ in $\left.\mathrm{H}_{2} \mathrm{O}\right)$ were neutralized with $N$-methyl-D-glucamine. APV was stored as $100 \mathrm{~mm}$ stock neutralized with $\mathrm{NaOH}$. All stock solutions were stored at $-20^{\circ} \mathrm{C}$. For measurements NFR was further supplemented with $30 \mu \mathrm{M}$ glycine (for glutamate dose-response measurements), or with $30 \mu \mathrm{M}$ to $1 \mathrm{~mm}$ glutamate, depending on the glutamate potency of each receptor (for glycine doseresponse measurements).

Application of solutions was computer-controlled by the program EggWorks. Applications of agonist were kept as short as possible, consistent with reaching a plateau or peak response. Data were recorded simultaneously on a chart recorder and on a computer hard disk. For digital recording, data were filtered at $100 \mathrm{~Hz}$ digitized at $300 \mathrm{~Hz}$ and reduced to a final sampling rate of $10 \mathrm{~Hz}$ (oversampling with equal weight averaging of 30 samples) before storage.

Data analysis for dose-response curves. Dose-response curves were fitted individually for each oocyte with the Hill equation:

$$
I=I_{\max } /\left(1+\left(\mathrm{EC}_{50} /[\mathrm{A}]\right)^{n \mathrm{H}}\right)
$$

where $n_{\mathrm{H}}$ is the Hill coefficient, $I_{\max }$ is the maximum current, [A] is the concentration of agonist, and $\mathrm{EC}_{50}$ is the concentration of agonist that produces a half-maximum response. Each data point was then normalized to the fitted maximum of the dose-response curve.

The normalized values were then pooled and averaged for each mutant and fitted again with the Hill equation.

Schild analysis. APV antagonism was examined by the Schild method (Arunlakshana and Schild, 1959). Recording conditions were as described above, with the exception that the perfusion solution contained $1.8 \mathrm{~mm}$ $\mathrm{BaCl}_{2}$. Oocytes were perfused for $60 \mathrm{sec}$ with APV-supplemented perfusion solution before application of glutamate in the presence of APV. Dose ratios from individual oocytes were determined at low agonist concentrations as follows. The currents induced by two concentrations $\left(\ll \mathrm{EC}_{50}\right)$ of agonist were determined in the absence of APV. Responses in the presence of a series of APV concentrations were then obtained with glutamate concentrations increased to produce similar responses.

The resulting set of two-point dose-response curves was fitted with the low-concentration limit of the Hill equation, i.e., with the power function $y=\left(x / x_{0}\right)^{\mathrm{n}}$. This gives straight lines on log-log coordinates, and the lines were constrained to be parallel by fitting the same $n$ for all curves. The dose ratios $(r$, defined as the ratio of the glutamate concentrations needed to produce the same response in the presence and absence of APV) were obtained for each APV concentration from this fit. The mean dose ratios for each concentration of APV were used to construct a Schild plot, of $\log (r-1)$ versus $\log [\mathrm{B}]$ where [B] is the antagonist concentration. This was initially fitted with a straight line, not in logarithmic form, but by fitting the $(r-1)$ and [B] values with a power function as above. In this form $n$ is the slope of the Schild plot, which is predicted by the Schild equation to be unity for a competitive antagonist at equilibrium. If $n$ was sufficiently close to 1 , then the results were to be taken to be consistent with the Schild equation, so the data were refitted with the slope fixed at 1 , i.e., they were refitted with the Schild equation $(r-1)=[\mathrm{B}] / K_{\mathrm{B}}$ in which the only free parameter is the equilibrium constant for antagonist binding, $K_{\mathrm{B}}$.

Single-channel recordings. Oocytes with agonist-induced $I_{\max }>0.5 \mu \mathrm{A}$ at $-60 \mathrm{mV}$ were selected for single-channel recording. Before making patch-clamp recordings, the vitelline membrane of each oocyte was removed in a hypertonic solution containing (in $\mathrm{mM}$ ): sodium methylsulfate 200, $\mathrm{KCl} 20, \mathrm{MgCl}_{2}$, $\mathrm{HEPES} \mathrm{10,} \mathrm{HH} 7.4$, with $\mathrm{KOH}$. Recordings of channel activity in outside-out patches were made in an external solution containing (in mM): $\mathrm{NaCl} 125, \mathrm{KCl} 3, \mathrm{NaH}_{2} \mathrm{PO}_{4} 1.25, \mathrm{HEPES} 20, \mathrm{CaCl}_{2}$ 0.85 , pH 7.4, with $\mathrm{NaOH}$ supplemented with $20 \mu \mathrm{M}$ glycine. "Low" glutamate concentrations were used. Because of the low glutamate potency on the T671A mutant, a "low concentration" means 30-200 $\mu \mathrm{M}$, 1000 -fold larger than used for the wild type. Patch pipettes, made from thick-walled borosilicate glass (Clark Electromedical Instruments, Pangbourne, UK), contained (in $\mathrm{mm}$ ): potassium gluconate 141, $\mathrm{NaCl} 2.5$, HEPES 10, EGTA 11, pH 7.4, with KOH. After fire-polishing of their tips, pipettes had resistance of 10-20 $\mathrm{M} \Omega$. Single-channel currents were recorded with an Axopatch-1D amplifier (Axon Instruments, Foster City, CA) and stored on digital audio tape (Biological DTR 1200/1205). For subsequent analysis, data were filtered at $2 \mathrm{kHz}(-3 \mathrm{~dB})$ before digitization at $20 \mathrm{kHz}$. Channel durations and amplitudes were fitted with the SCAN program (Colquhoun and Sigworth, 1995) and subjected to a 70 $\mu$ sec imposed resolution. We define an open period as a period during which the channel appeared to be continuously open, regardless of transitions between different open levels. Chord conductance values assume a reversal potential of $0 \mathrm{mV}$, which is close to the observed reversal potential between 0 and $10 \mathrm{mV}$. Observed channel activity was blocked by the glycine site antagonist 7-chlorokynurenic acid (25-50 $\mu \mathrm{M})$.

Concentration jump experiments. Fast concentration jumps were achieved by the rapid switching, across the tip of a patch pipette, of two solutions flowing from either side of a piece of theta glass. The control solution was supplemented with $20 \mu \mathrm{M}$ glycine, and the test solution contained $10 \mathrm{~mm}$ glutamate $(+20 \mu \mathrm{M}$ glycine $)$. Outside-out patches, held at $-100 \mathrm{mV}$, were exposed to glutamate for $100 \mathrm{msec}$, and jumps were made every $10 \mathrm{sec}$. Measurement of liquid junction potentials at the end of experiments indicated that the exchange of control and test solutions occurred within $300 \mu \mathrm{sec}$.

\section{RESULTS \\ NR2A mutations}

Ten mutants containing single amino acid substitutions of the NR2A subunit were generated by site-directed mutagenesis, at positions that were chosen either because they control glycine potency on NR1 subunits, or because they control amino acid binding in bacterial binding proteins. Alignment of NR2 sequences with bacterial amino acid binding proteins (Fig. $1 A$ ) reveals limited linear sequence identity in segments $\mathrm{N}$ terminal of $\mathrm{M} 1$ and $\mathrm{C}$ terminal of M3, in extracellular domains referred to as S1 and S2, respectively (Stern-Bach et al., 1994). The corresponding segments on NR1 harbor residues that control glycine potency (Kuryatov et al., 1994). Mutants were generated according to the following rule: uncharged amino acids were mutated into alanine, positive charges were substituted by negative charges, and in addition aromatic residues were replaced by another aromatic residue.

Recombinant coexpression of NR1 cRNA with any of the 10 mutant NR2A cRNAs resulted in robust inward currents after the application of glutamate in the presence of glycine (Fig. 1B). Injection of similar amounts of cRNA resulted in maximal agonist-induced currents of comparable magnitude; in particular, $I_{\max }$ for the mutants was generally not lower than $I_{\max }$ for the wild type (the values are given in the legend of Fig. 2). 
A

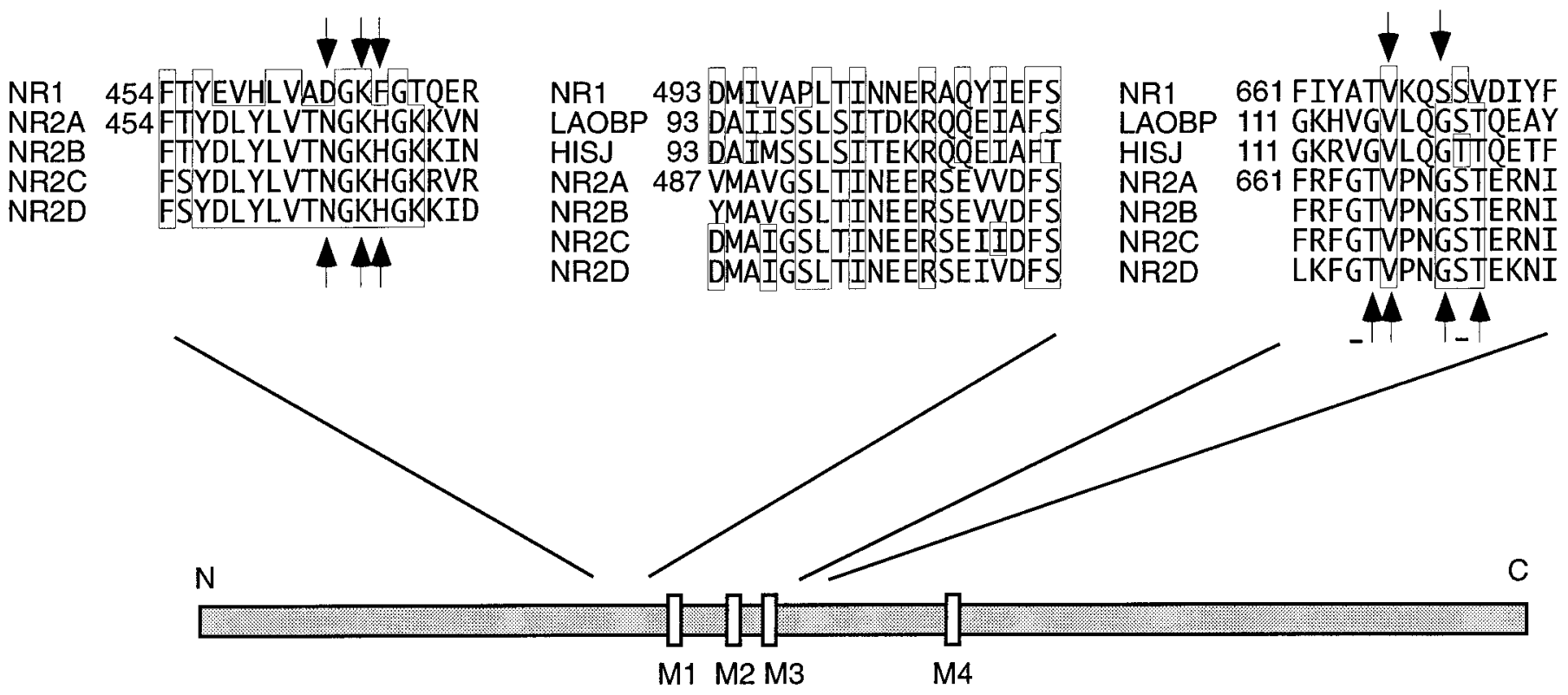

B

Wild-type

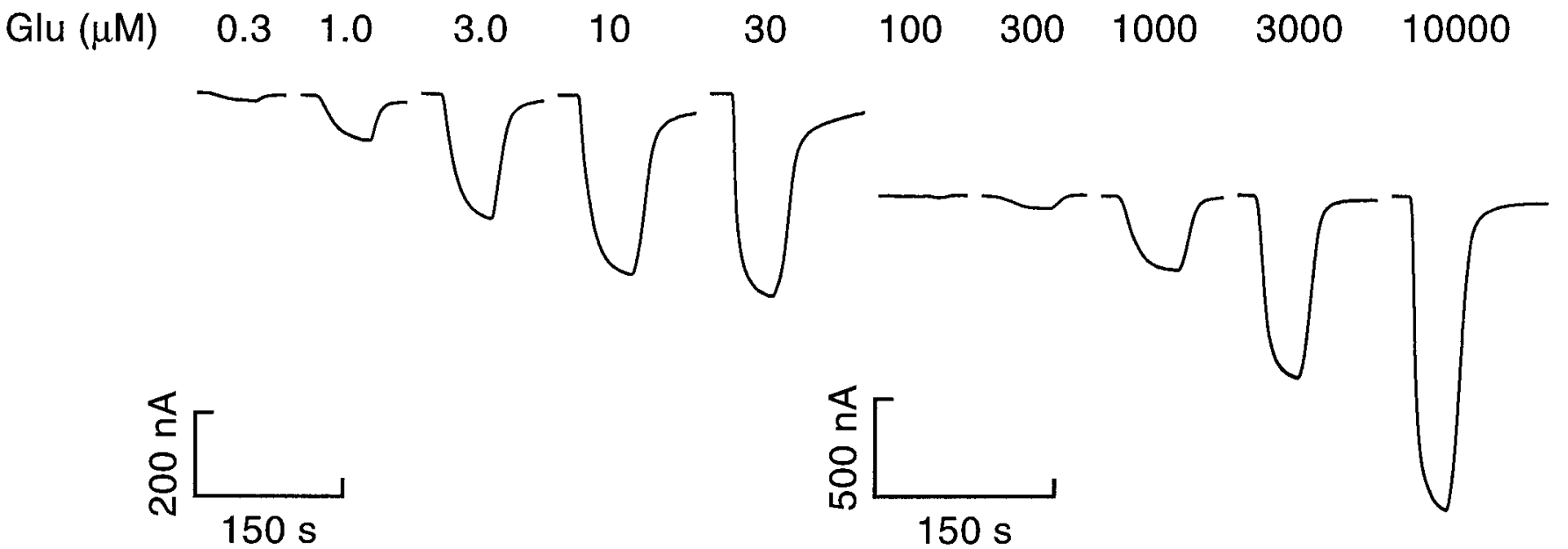

Figure 1. Single amino acid substitutions in the NR2A subunit generated functional NR1/NR2A receptors. $A$, Amino acid sequence alignment of rat NR1 (Moriyoshi et al., 1991) and NR2 subunits (Monyer et al., 1992; Ishii et al., 1993) with the bacterial periplasmic binding proteins LAOBP (Oh et al., 1993) and HISJ (Oh et al., 1994). Residues that reduced glycine potency when mutated in NR1 are marked by an arrow above the sequence alignment. NR2A residues that were mutated in this study are marked below the sequence alignments by an arrow if the mutation reduced potency by more than a factor of 2 ; otherwise they are indicated by a minus sign. Numbers indicate the first residue of the alignment; numbering is for mature proteins without signal peptides. $B$, Glutamate-activated currents recorded in the two-electrode voltage-clamp configuration from an oocyte expressing wild-type receptors (left) and an oocyte expressing T671A mutant receptors (right). Increasing concentrations of glutamate were applied in the presence of $30 \mu \mathrm{M}$ glycine.

\section{Reduced potency of glutamate}

Dose-response curves were recorded (Fig. 1B) in conditions (180 $\left.\mu \mathrm{M} \mathrm{Ba}^{2+}\right)$ designed to minimize the artifacts caused by agonistinduced influx of divalent cations (Leonard and Kelso, 1990; Williams, 1993). With the exception of NR2A(G664A), all of the mutations we tested shifted the dose-response curves for gluta- mate to the right (Fig. 2A). For all mutations except S670A (and G664A), the potency of glutamate was reduced by more than fivefold. Three mutations (H466A, G669A, and T671A) decreased the potency of glutamate by more than two orders of magnitude (Table 1). The fitted dose-response curves (Fig. 2A) show that the Hill coefficients for most of the 10 mutant receptors 


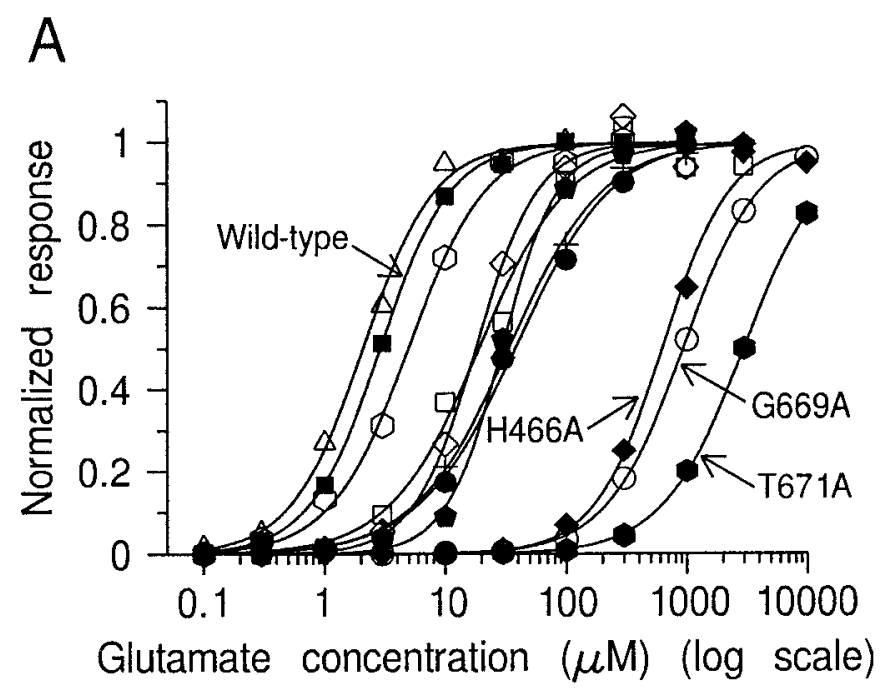

Figure 2. Mean normalized dose-response relationships for glutamate and glycine. $A$, Glutamate-activated currents were evoked in the presence of $30 \mu \mathrm{M}$ glycine. Mean dose-response curves for wild-type ( filled squares, mean of fitted $I_{\max }=0.41 \mu \mathrm{A}$ ), N463A (open diamonds, $1.3 \mu \mathrm{A}$ ), K465E ( filled pentagons, $1.6 \mu \mathrm{A}$ ), H466A ( filled diamonds, $1.4 \mu \mathrm{A}$ ), $\mathrm{H} 466 \mathrm{~F}$ ( filled circles, $1.8 \mu \mathrm{A}$ ), G664A (open triangles, $1.1 \mu \mathrm{A}$ ), T665A (open squares, 0.42 $\mu \mathrm{A})$, V666A (plus signs, $1.7 \mu \mathrm{A}), \mathrm{G} 669 \mathrm{~A}$ (open circles, $3.7 \mu \mathrm{A}), \mathrm{S} 670 \mathrm{~A}$ (open hexagons, $0.38 \mu \mathrm{A}$ ), and T671A ( filled hexagons, $2.0 \mu \mathrm{A}$ ) receptors were fitted with the Hill equation as described in Materials and Methods. Error bars have been omitted for clarity. The points represent the means of two to eight observations. $B$, Glycine-activated currents were evoked in the presence of a saturating concentration of glutamate $(30 \mu \mathrm{M}$ to $10 \mathrm{~mm}$ depending on glutamate potency for each receptor). Dose-response curves were constructed as in $A$. Symbols represent the same as in $A$.

are not much different from the value found for wild-type NR1/ NR2A receptors. The slopes for $\mathrm{H} 466 \mathrm{~F}$ and V666A are probably lower, and for K465E may be higher, than for the wild type, but the three mutations with the largest increase in $E C_{50}$ show little or no change of the Hill slope.

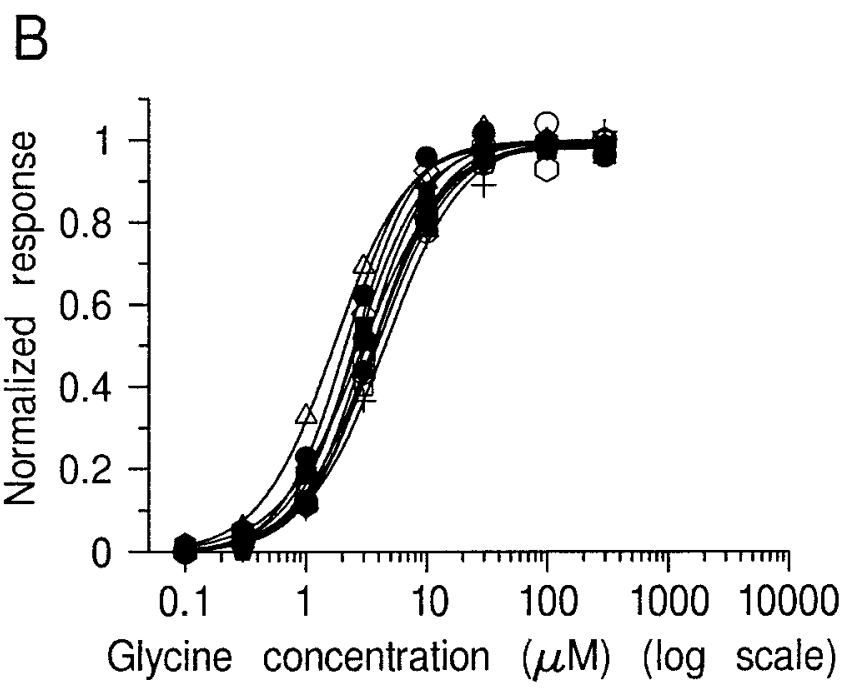

In contrast to the considerable effect of the mutations on glutamate potency, the potency of the coagonist glycine was barely affected; the $E C_{50}$ values for all 10 mutants lay within a factor of 1.7 of that of the wild type, as shown in Figure $2 B$.

Table 1. $\mathrm{EC}_{50}$ and Hill values for glutamate and glycine

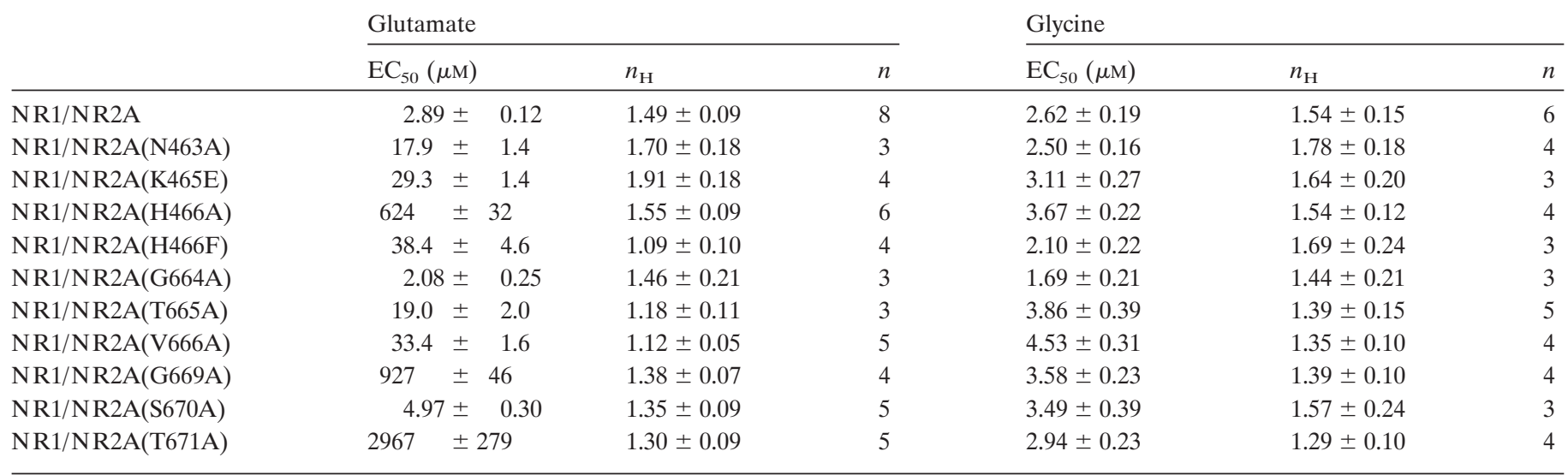



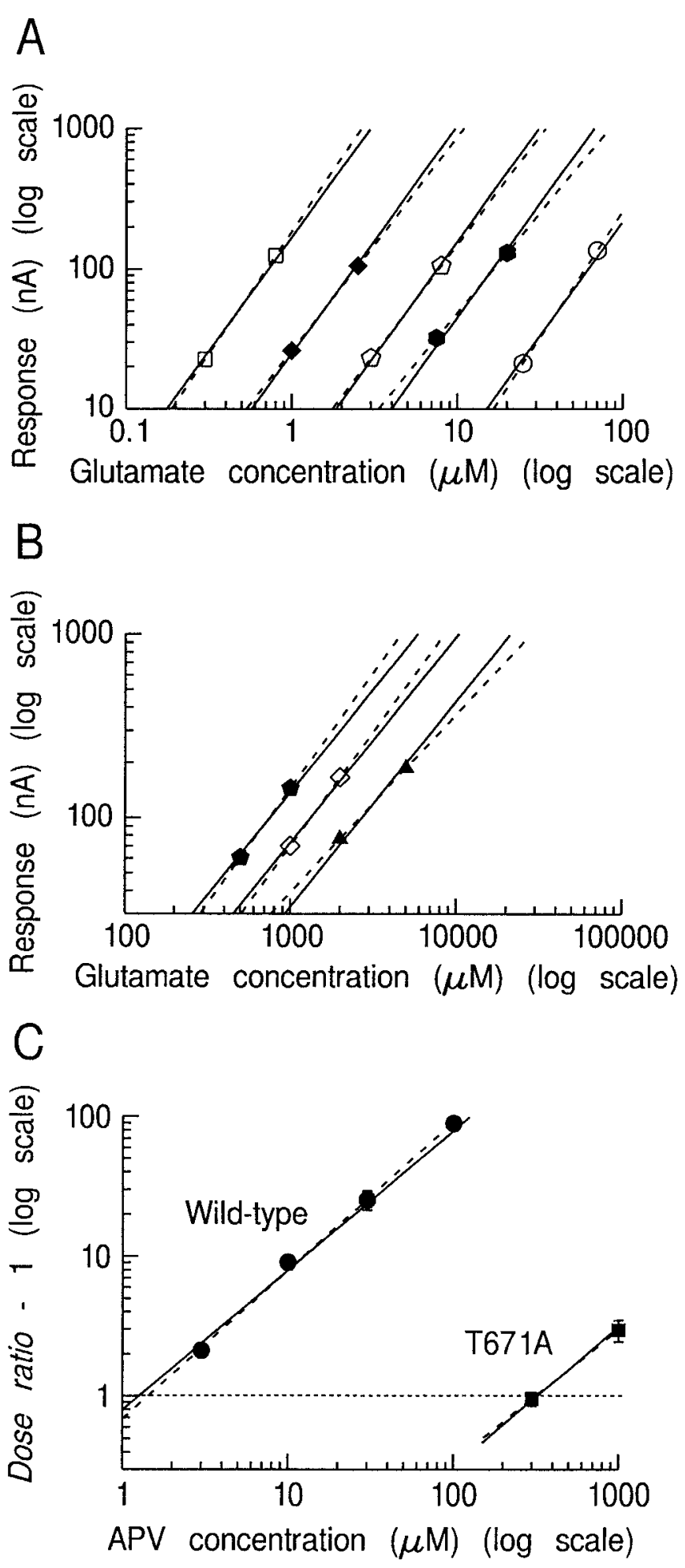

Figure 3. Schild analysis of the competitive antagonism of the glutamate binding site by APV. $A$, Partial, low-concentration, glutamate doseresponse curves for one oocyte expressing wild-type receptors in the absence (open squares) and presence of $3 \mu \mathrm{M}$ (filled diamonds), $10 \mu \mathrm{M}$ (open pentagons), $30 \mu \mathrm{M}$ ( filled hexagons), and $100 \mu \mathrm{M}$ (open circles) APV. Dashed lines represent free fits of the power function (see Materials and Methods) to the data, and solid lines show fits of the same function but with slopes constrained to be the same for all curves. Fitted lines have been extrapolated for display purposes. $B$, Partial, low-concentration, glutamate dose-response curves for one oocyte expressing T671A mutant receptors in the absence (filled pentagons) and presence of

\section{Reduced affinity for APV}

To examine the underlying mechanism that is responsible for the decreased potency of glutamate (see Discussion), we analyzed the NR1/NR2A(T671A) mutant in greater detail. Schild's method (Arunlakshana and Schild, 1959) was used to determine the equilibrium constant, $K_{\mathrm{B}}$, for the competitive antagonist APV (Fig. 3).

The slope of the Schild plot was $1.06 \pm 0.04$ for the wild type and $0.94 \pm 0.16$ for T671A. These values are close to (and do not differ significantly from) 1.0, so there is no evidence against the view that $\mathrm{APV}$ is a competitive antagonist on both receptors. Therefore the plots were refitted with the Schild equation (slope fixed at 1), as described in Materials and Methods, and these fits are shown in Figure $3 C$. For the wild type we found that $K_{\mathrm{B}}=1.26 \pm 0.07 \mu \mathrm{M}$, which is similar to the value of $1.35 \mu \mathrm{M}$ reported by Verdoorn et al. (1989) for rat brain mRNA expressed in oocytes. For the T671A mutant, however, we found a much reduced affinity for APV, with $K_{\mathrm{B}}=321 \pm 30 \mu \mathrm{M}$. This 255 -fold reduction in affinity is comparable with the 1000-fold reduction of potency for glutamate.

\section{Single-channel conductance}

The single-channel currents evoked by glutamate in NR1/ NR2A(T671A) were remarkably similar to those evoked in the wild type (Fig. 4). The mean chord conductances of their main level and sublevel were estimated as $51.1 \pm 1.1 \mathrm{pS}$ and $38.6 \pm 0.9$ $\mathrm{pS}(n=5)$, compared with values of $50.1 \pm 1.4 \mathrm{pS}$ and $38.3 \pm 1.3$ pS reported for wild-type NR1/NR2A by Stern et al. (1992). The relative areas for these two components, $85 \%$ and $15 \%$, were also similar to those for wild type ( $80 \%$ and $20 \%)$. In addition, the frequencies of direct transitions from shut to main level, from shut to sublevel, and from main to sublevel of T671A channels (Table 2) were similar to those reported for the wild type by Stern et al. (1992). Like the wild-type NR1/NR2A, they showed no sign at all of the sort of temporal asymmetry shown by NR1/NR2D receptors (Wyllie et al., 1996).

\section{Single-channel kinetics}

In contrast to the close similarity of the conductances, there were kinetic differences between T671A and the wild type. For example, both mutant and wild-type open period distributions contained three exponential components (Fig. 5). The mean time constants for each component were $66.2 \pm 17.0 \mu \mathrm{sec}, 1.10 \pm 0.34$ msec, and $6.91 \pm 2.45 \mathrm{msec}$ for T671A channels $(n=5)$, and $47.6 \pm 9.3 \mu \mathrm{sec}, 0.823 \pm 0.375 \mathrm{msec}$, and $3.08 \pm 0.57 \mathrm{msec}$ for wild-type channels $(n=5)$ (P. Béhé and D. Wyllie, personal communication). The overall mean apparent length of the open periods was $4.33 \pm 0.71 \mathrm{msec}$ for T671A compared with $1.69 \pm$ $0.16 \mathrm{msec}$ for the wild type (also see Fig. 4). These values are clearly different ( $p=0.0045$ by two-tailed randomization test).

For this comparison, both wild-type and mutant data were analyzed with the same resolution (70 $\mu$ sec for both open and shut times). Such a resolution would mean that a substantial number of brief shuttings were missed; however, it is not possible to do a satisfactory correction for missed events without knowledge of a realistic kinetic mechanism (Colquhoun and Hawkes, 1995).

$300 \mu \mathrm{M}$ (open diamonds) and $1 \mathrm{mM}$ ( filled triangles) APV. Data were fitted as in $A$. C, Schild plot for antagonism of wild-type (filled circles) and T671A ( filled squares) receptors by APV using dose ratios estimated from results such as those in $A$ and $B$. The points represent the means of five dose ratios. The dashed lines are free fits with slopes that are not exactly 1; the solid lines are fits of the Schild equation (both have slope =1). Fitted lines have been extrapolated for display purposes. 
A

T671A
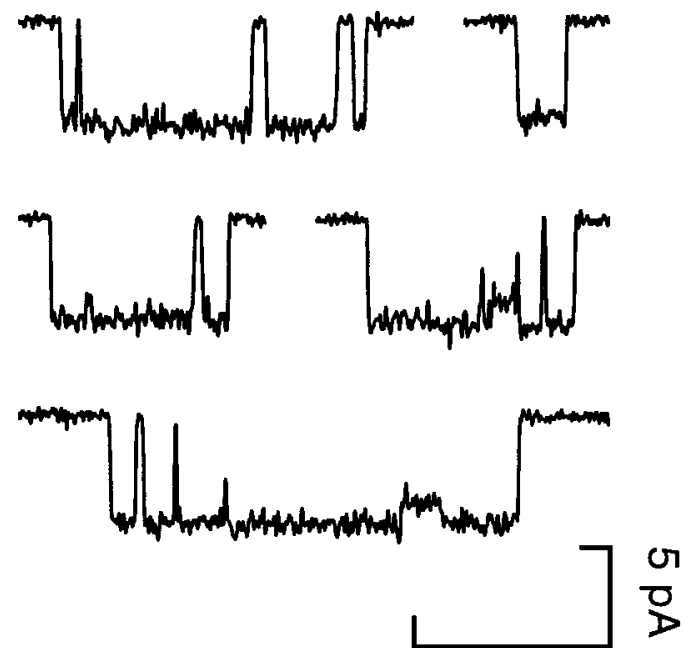

C

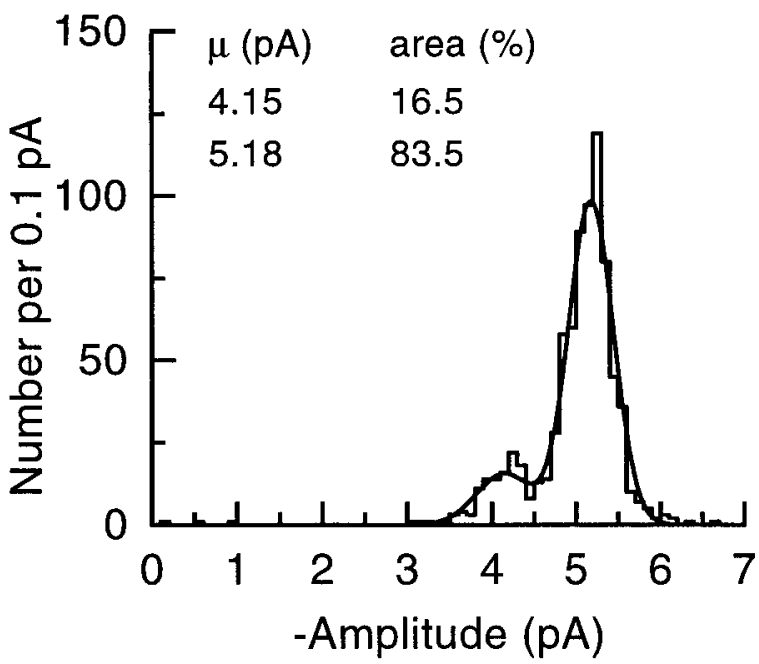

$\mathrm{B}$ Wild-type
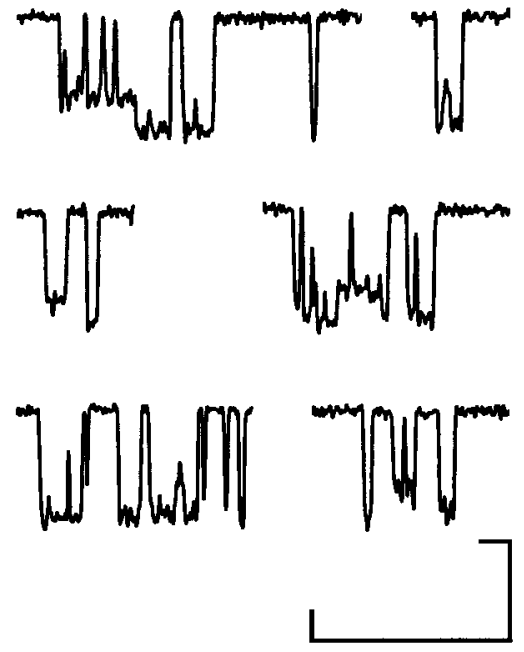

$\stackrel{0}{0}$
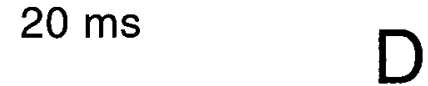

$20 \mathrm{~ms}$

Figure 4. Wild-type and T671A single-channel amplitudes. $A$, Single-channel currents recorded in an outside-out patch from an oocyte expressing T671A mutant receptors in the presence of $60 \mu \mathrm{M}$ glutamate and $20 \mu \mathrm{M}$ glycine. At $-100 \mathrm{mV}$ a main level $(\sim 5 \mathrm{pA})$ and a sublevel $(\sim 4 \mathrm{pA})$ are clearly visible. $B$, Wild-type single-channel currents adapted from Béhé et al. (1995). Transitions between the main level $(\sim 5 \mathrm{pA})$ and sublevel $(\sim 4 \mathrm{pA})$ are evident. $C$, Amplitude histogram of 791 events of duration greater than 2.5 filter rise times, for the patch illustrated in $A$. The distribution was fitted with two Gaussian components with means of 5.18 and $4.15 \mathrm{pA} D$, Amplitude histogram for the same patch as in $B$ reanalyzed from Béhé et al. (1995). The distribution of 1048 events greater than 2.5 rise times was fitted with two Gaussian components with means of 5.52 and 4.35 pA.

Kinetic differences between mutant and wild-type receptors were also observed in concentration jump experiments. Preliminary data, shown in Figure 6, indicate that deactivation of T671A receptors, after a $100 \mathrm{msec}$ jump of $10 \mathrm{~mm}$ glutamate, occurs with a time constant of $18.2 \pm 3.0 \mathrm{msec}(n=4)$. This is approximately one order of magnitude faster than that found for the deactivation of wild-type NR1/NR2A channels after "long" pulses of glutamate (Monyer et al., 1992; Medina et al., 1995) (D. Wyllie and P. Béhé, personal communication).

\section{DISCUSSION}

\section{Glutamate effects are mediated through NR2 subunits}

We have shown that mutagenesis of NR2A residues reduced the potency of glutamate by up to 1000 -fold, with very little effect on
Table 2. Transitions between conductance levels for NR1/NR2A(T671A) channels

\begin{tabular}{lrr} 
Transition type & $n(N=10897)^{a}$ & \multicolumn{1}{c}{$\%$} \\
\hline Shut-sublevel & 400 & 3.67 \\
Sublevel-shut & 366 & 3.36 \\
Shut-main level & 4210 & 38.63 \\
Main level-shut & 4241 & 38.92 \\
Sublevel-main level & 860 & 7.89 \\
Main level-shut & 820 & 7.53
\end{tabular}

Experiments were performed in the presence of $20 \mu \mathrm{M}$ glycine and between 60 and $200 \mu \mathrm{M}$ glutamate.

${ }^{a} N$ refers to the total number of transitions, longer than $2.5 t_{\mathrm{r}}$, analyzed from five recordings. 


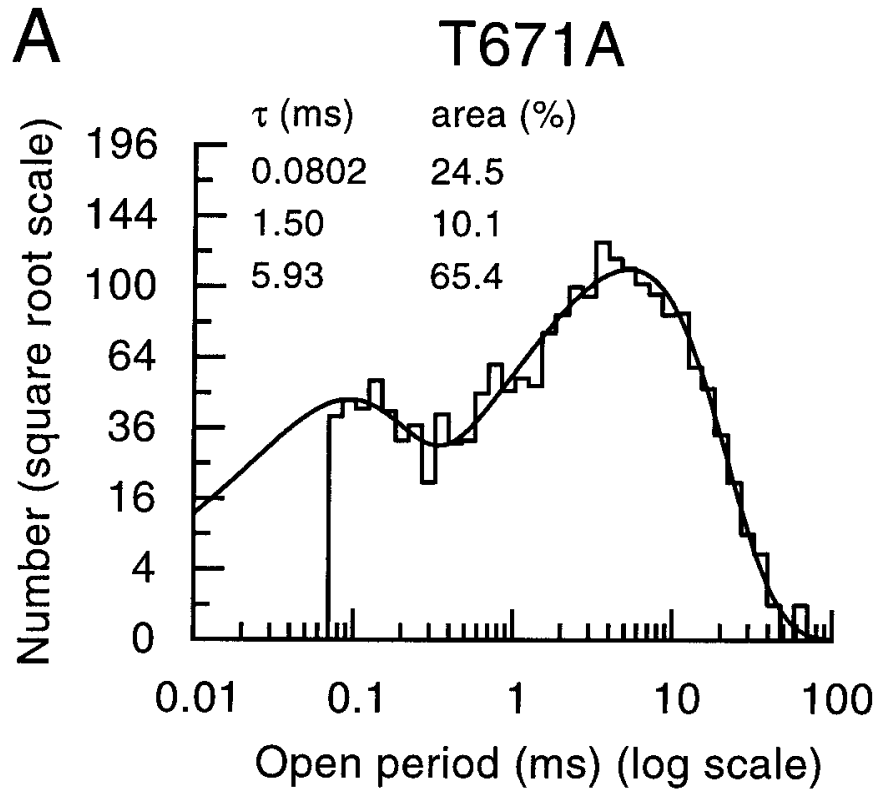

B Wild-type

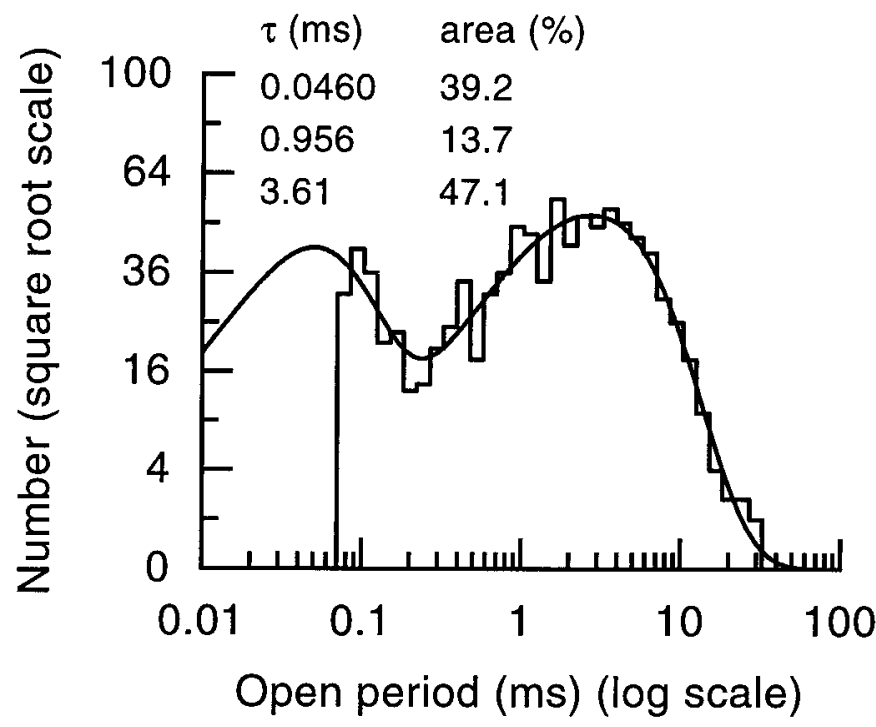

Figure 5. Open period distribution for T671A mutant and wild-type channels. $A$, Distribution of the length of 1947 apparent open periods evoked by $200 \mu \mathrm{M}$ glutamate and $20 \mu \mathrm{M}$ glycine in a patch containing T671A mutant channels. The distribution was fitted with a mixture of three exponential densities with parameters as shown. B, Distribution of 949 apparent open periods evoked by $30 \mathrm{~nm}$ glutamate $(+20 \mu \mathrm{M}$ glycine $)$ in a patch excised from an oocyte expressing wild-type NR1/NR2A receptors. The distribution was fitted with a mixture of three exponential components.

the potency of glycine. Our evidence strongly suggests (see below) that the binding site for glutamate on NMDA NR1/NR2A receptors is formed by NR2A subunits. The residues we found to be important in NR2A are fully conserved within the NR2 family (Fig. $1 A$ ), which suggests that in all types of NR1/NR2 NMDA receptors glutamate effects are mediated by the NR2 subunit. Comparison of our data with the results of Kuryatov et al. (1994) and Wafford et al. (1995) on glycine potency of NR1 mutations suggests that the glutamate effects mediated through the NR2 subunits, and glycine effects mediated through the NR1 subunit, are each based on the same structural design.

While the experiments described here were in progress, Laube et al. (1997) reported that mutation of NR2B residues reduced glutamate potency on NR1/NR2B receptors. Three of their mutations were equivalent to ours; for two of them we both found a comparable (approximately 10-fold) reduction in potency of glutamate (H466F, V666A), whereas for one (K465E, equivalent to NR2B K459E) we found a considerably smaller effect. For NR2A S670, we analyzed the effect of the mutation to alanine, S670A, and found only a minimal effect; the same serine to alanine mutation at the equivalent position on the NR1 subunit also has little effect on glycine potency (Kuryatov et al., 1994). In contrast, Laube et al. (1997) report a 180-fold reduction of glutamate potency for a mutation into glycine at the homologous position (S664G). The mutations that gave the three largest increases in glutamate $E C_{50}$ in Laube et al. (1997) (236-, 180-, and 118 -fold) were all at different positions from those that gave the largest shifts here (1027-, 321-, and 216-fold). Furthermore their mutant with the largest shift actually showed a decreased $I C_{50}$ for APV, although that with the 180 -fold shift showed a 40 -fold increase in $I C_{50}$. Our most-shifted mutant, T671A, showed a 255-fold increase in the equilibrium binding constant of APV. Laube et al. (1997) proposed an appealing three-dimensional arrangement for the structure of the glutamate binding site, and it will be interesting to see how this proposal can accommodate the rather different residues that seem, from the mutants tested here, to be important for glutamate binding.

\section{Evidence that reduced potency is caused by impaired binding of glutamate}

The basis for the inference that the mutated residues form part of the glutamate binding site requires clarification. A reduction of potency is not necessarily caused by a change in the binding site but can also be caused by a change of gating. In other words, the glutamate binding site might not be affected at all; furthermore, a binding assay for glutamate would not resolve this ambiguity (see Colquhoun and Farrant, 1993).

To infer that the mutated residues form part of the binding site it is necessary to show that the mutation has altered the microscopic equilibrium constant for the initial binding step: the binding of glutamate to the resting state of the receptor. If this were the only change we could say that it was very likely that these domains form part of the glutamate binding site (we do not distinguish in this paper between residues that interact directly with the ligand and residues that shape the binding site without direct interaction with the ligand).

One way to examine this problem is to use a competitive antagonist rather than an agonist. This removes most of the complications in interpretation of the effect of a mutation, because a competitive antagonist, unlike an agonist, is not expected to cause a change of the receptor conformation to higher-affinity active states (i.e., gating). Thus any change in their binding is likely to result directly from a change in the binding site. The equilibrium constant for antagonist binding, $K_{\mathrm{B}}$, cannot be determined from $I C_{50}$ measurements, so Schild's method was used. It is possible that binding of the agonist might be altered in a mutant receptor without alteration of binding of a competitive antagonist, because the binding sites for the two compounds, although presumably overlapping, are not identical. However, we found 

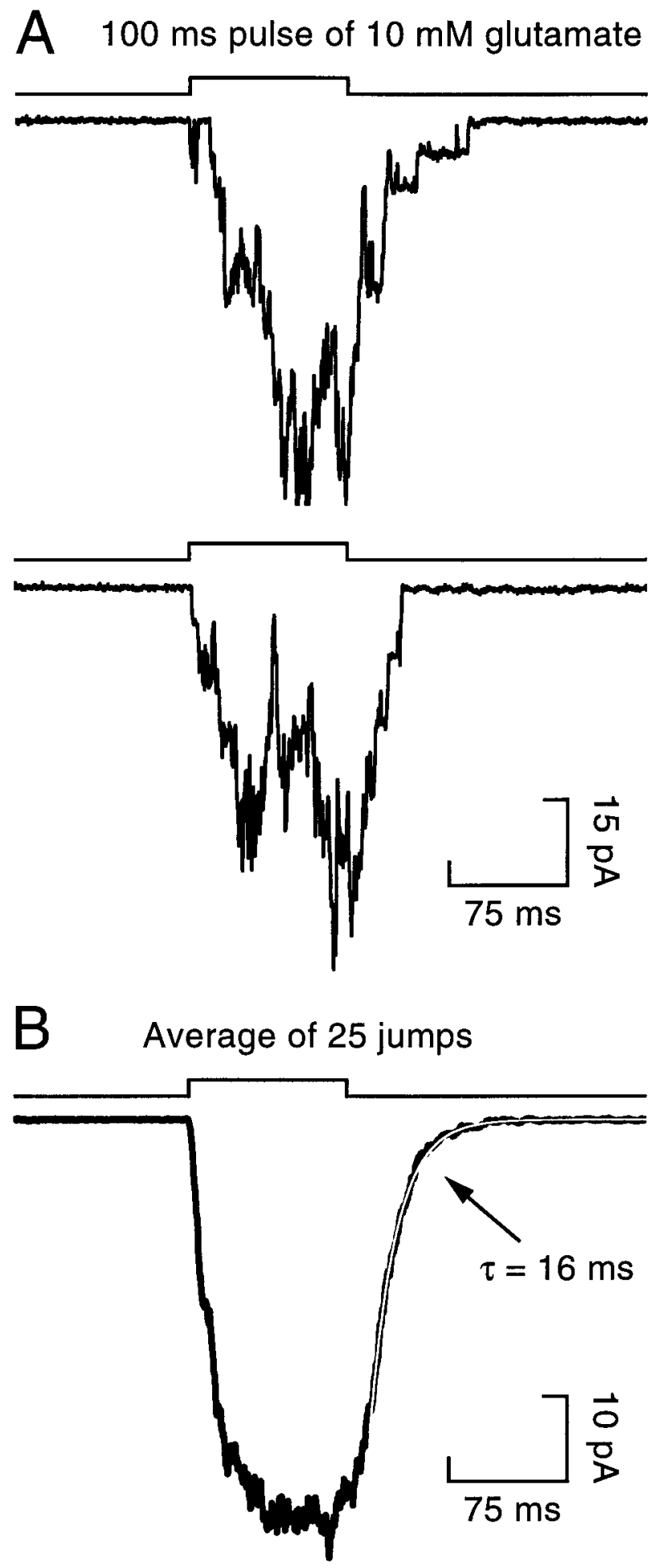

Figure 6. Fast concentration jumps on the T671A mutant receptor. $A$, Two individual responses obtained from an outside-out patch after a 100 msec pulse of $10 \mathrm{~mm}$ glutamate (in the presence of $20 \mu \mathrm{M}$ glycine). Single-channel currents can be seen clearly in these example traces. The timing and duration of the glutamate application are indicated above each pulse. $B$, The average of 25 such jumps. The deactivation of this ensemble current is rapid and can be reasonably well described by a single exponential with a time constant of $16 \mathrm{msec}$, shown as a white line. that the T671A mutation increased the equilibrium binding constant 255-fold for APV compared with wild type. Therefore in this mutant, APV binding is impaired greatly, which implies that its binding site, and therefore probably that for glutamate, has been altered.

The equilibrium concentration-response curves also suggest a primary effect of the mutation on the binding site for glutamate. The main finding is that mutations in the NR2 subunit can produce an enormous reduction in the potency of glutamate $\left(E C_{50}\right)$, with little change in the Hill slope, and very little change in the potency of glycine (Table 1). Of the three most effective mutations, one precedes M1 (S1 domain) and the others follow M3 (S2 domain), which suggests that both regions contribute to the binding site.

A reduction in the open-shut equilibrium constant could produce this result without any detectable change in maximum response, provided that its reduced value is still such that most channels are open at equilibrium. How can we exclude this possibility? The first reason is that for many cooperative mechanisms we expect the shift of the $E C_{50}$ to be proportional to the binding constants but roughly proportional (in the case of two bindings being required) to the square root of the gating constant (as long as this equilibrium is well over toward the open state). In this case the 1000 -fold increase in $E C_{50}$ seen with T671A would require a roughly millionfold reduction in the gating constant. If this were to occur without a drastic reduction of the maximum response, the gating constant for the wild-type channel would have to be enormously high. This does not appear to be the case, because glutamate actually behaves like a partial agonist on NMDA receptors with a maximum $P_{\text {open }}$ of $\sim 0.3$ (Gibb and Colquhoun, 1992; Dzubay and Jahr, 1996). Therefore, such a reduction in the gating constant would cause an enormous reduction in the maximum response. In common with almost every mutation study, we have no good evidence as to whether the maximum response is changed, because of uncertainties about the relative efficiency of expression of wild-type and mutant RNAs; however, there was no systematic difference between the maxima observed (Fig. 2 legend), so it is clear that changes by a factor of one million have not occurred.

Furthermore, most kinetic mechanisms predict that the Hill slope would be reduced substantially if there were such a large effect on the gating constant as would be required to account for our observations; we observed no such reduced Hill slope.

\section{Interpretation of kinetic data of the T671A mutant}

In principle, effects on channel gating can be measured from kinetic analysis of single-channel recordings (Colquhoun and Sakmann, 1985). We show that transition frequencies between the conductance levels of T671A (as well as the levels themselves) are similar to those of the wild type. Therefore, at least this aspect of gating is not altered in the mutant with the largest reduction in potency.

The observation that the mean apparent open period is longer for the T671A mutant could mean that the channels stay open rather longer, which would imply some effect on the shutting rate of the channel, i.e., on the gating process. Such a change, however, is in the wrong direction to explain reduced glutamate potency. In any case it is quite conceivable that there is no real increase in the length of individual openings; the apparent increase could well result from more rapid dissociation of the agonist, which would be expected to reduce the duration and frequency of brief shuttings such that fewer of them are detected and openings appear, incorrectly, to be longer. 
Figure 6 shows that the decay of the response, after rapid removal of glutamate, is roughly 10 -fold faster for T671A than for wild-type receptors. The decay rate of the current is a reflection of the length of the underlying channel activation, or burst (Colquhoun et al., 1997). We have found that the individual openings, if anything, are longer in the mutant receptor, so the fast decay cannot be explained by shorter open times. The length of an activation will depend on the number of times that the channel reopens while agonist is still bound. The more rapid decay is therefore likely to result from there being fewer reopenings during an activation for the mutant channel compared with the wild type. This could result from either (1) an increased rate of dissociation of glutamate or (2) a reduced opening rate constant for the mutant channel. If we are right in assuming that wild-type and mutant receptors express with comparable efficiency, then, as discussed above, it is unlikely that the opening rate constant is greatly reduced in the mutant, so this is also unlikely to be the main reason for the faster decay (although a contribution from this source cannot be ruled out). Thus the main reason is likely to be that glutamate dissociates more rapidly, thereby reducing the probability that the channel will reopen. An increase in dissociation rate is consistent with a reduced affinity for glutamate.

\section{Implications}

In the absence of any completely satisfactory kinetic mechanism for the NMDA receptor, it is not possible to infer from the results, in a rigorous manner, numerical values for the microscopic equilibrium constant for the initial binding step or for the gating constants. As discussed above, it is most likely that the primary effect, at least for the three most effective mutations, is on glutamate binding.

The model of a conserved structural design of agonist binding sites of the extended ionotropic glutamate receptor family is further supported by other studies. Paas et al. (1996) investigated ligand binding to chick cerebellar kainate binding protein, and Kuusinen et al. (1995) found ligand binding by a recombinant fusion protein consisting only of fused S1 and S2 domains of an AMPA receptor.

\section{REFERENCES}

Arunlakshana O, Schild HO (1959) Some quantitative uses of drug antagonists. Br J Pharmacol Chemother 14:48-57.

Béhé P, Stern P, Wyllie DJ, Nassar M, Schoepfer R, Colquhoun D (1995) Determination of NMDA NR1 subunit copy number in recombinant NMDA receptors. Proc R Soc Lond [Biol] 262:205-213.

Choi DW (1988) Glutamate neurotoxicity and diseases of the nervous system. Neuron 1:623-634.

Collingridge GL, Bliss TV (1995) Memories of NMDA receptors and LTP. Trends Neurosci 18:54-56.

Colquhoun D, Farrant M (1993) The binding issue. Nature 366:510-511.

Colquhoum D, Hawkes AG (1995) The principles of the stochastic interpretation of ion channel mechanisms. In: Single Channel Recording, (Sakmann B, Neher E, eds), pp 397-482. New York: Plenum.

Colquhoun D, Sakmann B (1985) Fast events in single-channel currents activated by acetylcholine and its analogues at the frog muscle endplate. J Physiol (Lond) 369:501-557.

Colquhoun D, Sigworth FJ (1995) Fitting and statistical analysis of single-channel records. In: Single channel recording (Sakmann B, Neher E, eds), pp 483-587. New York: Plenum.

Colquhoun D, Hawkes AG, Merlushkin A, Edmonds B (1997) Properties of single ion channel currents elicited by a pulse of agonist concentration or voltage. Philos Trans R Soc Lond [A] 355:1743-1786.

Dzubay JA, Jahr CE (1996) Kinetics of NMDA channel opening. J Neurosci 16:4129-4134.

Gibb AJ, Colquhoun D (1992) Activation of $N$-methyl-D-aspartate receptors by L-glutamate in cells dissociated from adult rat hippocampus. J Physiol (Lond) 456:143-179.
Hirai H, Kirsch J, Laube B, Betz H, Kuhse J (1996) The glycine binding site of the $N$-methyl-D-aspartate receptor subunit NR1: identification of novel determinants of co-agonist potentiation in the extracellular M3-M4 loop region. Proc Natl Acad Sci USA 93:6031-6036.

Hollmann M, Heinemann S (1994) Cloned glutamate receptors. Annu Rev Neurosci 17:31-108.

Ishii T, Moriyoshi K, Sugihara H, Sakurada K, Kadotani H, Yokoi M, Akazawa C, Shigemoto R, Mizuno N, Masu M, Nakanishi S (1993) Molecular characterization of the family of the $N$-methyl-D-aspartate receptor subunits. J Biol Chem 268:2836-2843.

Kuner T, Schoepfer R (1996) Multiple structural elements determine subunit specificity of $\mathrm{Mg}^{2+}$ block in NMDA receptor channels. J Neurosci 16:3549-3558.

Kuryatov A, Laube B, Betz H, Kuhse J (1994) Mutational analysis of the glycine-binding site of the NMDA receptor: structural similarity with bacterial amino acid-binding proteins. Neuron 12:1291-1300.

Kuusinen A, Arvola M, Keinänen,K (1995) Molecular dissection of the agonist binding site of an AMPA receptor. EMBO J 14:6327-6332.

Laube B, Hirai H, Sturgess M, Betz H, Kuhse J (1997) Molecular determinants of agonist discrimination by NMDA receptor subunits: analysis of the glutamate binding site on the NR2B subunit. Neuron 18:493-503.

Leonard JP, Kelso SR (1990) Apparent desensitization of NMDA responses in Xenopus oocytes involves calcium-dependent chloride current. Neuron 4:53-60.

McBain CJ, Mayer ML (1994) N-methyl-D-aspartic acid receptor structure and function. Physiol Rev 74:723-760.

Medina I, Filippova N, Charlton G, Rougeole S, Ben-Ari Y, Khrestchatisky M, Bregestovski P (1995) Calcium-dependent inactivation of heteromeric NMDA receptor-channels expressed in human embryonic kidney cells. J Physiol (Lond) 482:567-573.

Monyer H, Sprengel R, Schoepfer R, Herb A, Higuchi M, Lomeli H, Burnashev N, Sakmann B, Seeburg PH (1992) Heteromeric NMDA receptors: molecular and functional distinction of subtypes. Science 256:1217-1221.

Moriyoshi K, Masu M, Ishii T, Shigemoto R, Mizuno N, Nakanishi S (1991) Molecular cloning and characterization of the rat NMDA receptor. Nature 354:31-37.

Nakanishi S (1992) Molecular diversity of glutamate receptors and implications for brain function. Science 258:597-603.

Oh BH, Pandit J, Kang CH, Nikaido K, Gokcen S, Ames GF, Kim SH (1993) Three-dimensional structures of the periplasmic lysine/arginine/ ornithine-binding protein with and without a ligand. J Biol Chem 268:11348-11355.

Oh BH, Kang CH, De Bondt H, Kim SH, Nikaido K, Joshi AK, Ames GF (1994) The bacterial periplasmic histidine-binding protein. Structure/ function analysis of the ligand-binding site and comparison with related proteins. J Biol Chem 269:4135-4143.

Paas Y, Eisenstein M, Medevielle F, Teichberg VI, Devillers-Thiéry A (1996) Identification of the amino acid subsets accounting for the ligand binding specificity of a glutamate receptor. Neuron 17:979-990.

Stern P, Béhé P, Schoepfer R, Colquhoun D (1992) Single-channel conductances of NMDA receptors expressed from cloned cDNAs: comparison with native receptors. Proc R Soc Lond [Biol] 250:271-277.

Stern-Bach Y, Bettler B, Hartley M, Sheppard PO, O'Hara PJ, Heinemann SF (1994) Agonist selectivity of glutamate receptors is specified by two domains structurally related to bacterial amino acid-binding proteins. Neuron 13:1345-1357.

Verdoorn TA, Kleckner NW, Dingledine R (1989) N-methyl-D-aspartate/glycine and quisqualate/kainate receptors expressed in Xenopus oocytes: antagonist pharmacology. Mol Pharmacol 35:360-368.

Wafford KA, Kathoria M, Bain CJ, Marshall G, Le Bourdellès B, Kemp JA, Whiting PJ (1995) Identification of amino acids in the $N$-methylD-aspartate receptor NR1 subunit that contribute to the glycine binding site. Mol Pharmacol 47:374-380.

Williams K (1993) Ifenprodil discriminates subtypes of the $N$-methyl-Daspartate receptor: selectivity and mechanisms at recombinant heteromeric receptors. Mol Pharmacol 44:851-859.

Williams K, Chao J, Kashiwagi K, Masuko T, Igarashi K (1996) Activation of $N$-methyl-D-aspartate receptors by glycine: role of an aspartate residue in the M3-M4 loop of the NR1 subunit. Mol Pharmacol 50:701-708.

Wyllie DJA, Béhé P, Nassar M, Schoepfer R, Colquhoun D (1996) Single-channel currents from recombinant NMDA NR1a/NR2D receptors expressed in Xenopus oocytes. Proc R Soc Lond [Biol] 263:1079-1086. 